Goldschmidt 2021 Abstract

https://doi.org/10.7185/gold2021.6298

\title{
Oxygenic origin of photosynthesis
}

\section{TANAI CARDONA}

Imperial College London

Presenting Author: t.cardona@imperial.ac.uk

The origin of oxygenic photosynthesis is considered a turning point in the history of life, marking the transition from the ancient world of anaerobes into a productive aerobic world that permitted the emergence of complex life. Today, it is accepted that photosynthesis originated in a bacterium at a late stage after the origin of life. It is also accepted that at the beginning photosynthesis was anoxygenic, and that it could have taken several hundreds of millions of years for oxygenic photosynthesis to emerge in [an ancestor of] cyanobacteria. In this talk, I will attempt to demonstrate how this view is constructed on historic assumptions that are not only unproven [1], but also not supported by the evolution of the key enzymes of photosynthesis, the photosystems.

Oxygenic photosynthesis starts with the oxidation of water to $\mathrm{O}_{2}$, a light-driven reaction catalysed by photosystem II. How and when exactly water oxidation originated have remained unanswered questions. I will provide a detailed account of the origin and molecular evolution of photosystem II and water oxidation as a function of time. I will show that: 1) the origin of a water-splitting photosystem can comfortably antedate the most recent common ancestor of cyanobacteria by over a billion years [2]; 2) photosystem II features patterns of molecular evolution that are indistinguishable from enzymes known to be as old as life itself [3]; and 3) the origin of photosystems likely occurred before the diversification of bacteria and in the context of the establishment of water oxidation [3].

Finally, I will conclude my talk by demonstrating that an oxygenic origin is a much simpler staring hypothesis for the study of the evolution of photosynthesis, with more explanatory and predictive power than the current paradigm.

1. Cardona, T., Thinking twice about the evolution of photosynthesis. Open Biology, 2019. 9: 180246. DOI: 10.1098/rsob.180246.

2. Cardona, T., P. Sánchez-Baracaldo, A.W. Rutherford, and A.W.D. Larkum, Early Archean origin of Photosystem II. Geobiology, 2019. 17: 127-150. DOI: 10.1111/gbi.12322.

3. Oliver, T., P. Sánchez-Baracaldo, A.W. Larkum, A.W. Rutherford, and T. Cardona, Time-resolved comparative molecular evolution of oxygenic photosynthesis. Biochim. Biophys. Acta, 2021: 148400. DOI: 10.1016/j.bbabio.2021.148400. 